\title{
Finite element optimization by pulsed thermography with adaptive response surfaces.
}

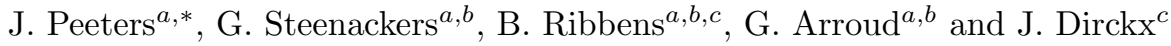 \\ ${ }^{a}$ University of Antwerp, Op3Mech research group, Faculty of applied engineering, Department of \\ Electromechanics, Salesianenlaan 30, B-2660 Antwerp, Belgium; \\ ${ }^{b}$ Vrije Universiteit Brussel (VUB), Department of Mechanical Engineering (MECH), Acoustics \& \\ Vibration Research Group (AVRG), Pleinlaan 2, B-1050, Brussels, Belgium; \\ ${ }^{c}$ University of Antwerp, Laboratory of Biomedical Physics (Bimef), B-2000 Antwerp, Belgium.
}

\begin{abstract}
A finite element (FE) model is updated and optimized by making use of the surface temperature distribution captured with pulsed thermography (PT) on a PVC plate. The temperature distribution across the surface can be measured. As the amount of experimental data to be used in this case is very high, an accurate, time-efficient adaptive response surface optimization algorithm is developed. The FE model will become more accurate and the simulations more realistic. The major advantages of using PT are that one picture contains the complete structure temperature data and that it is a non-contact technique.
\end{abstract}

Keywords: finite element model, pulsed thermography, optimization, Inverse Problem.

\section{Introduction}

The study of Pulsed Infrared Thermography (PIRT) has become an important aspect of Non-destructive Evaluation Techniques (NDE) for damage detection in metallic structural elements [1], as well as CFRP (Carbon Fibre Reinforced Polymer) and GFRP (Glass Fibre Reinforced Polymer) composites [2]. There are many advantages of thermography, these include the fact that no coupling is required, it can be performed in-situ, it can cover large areas and it is a quantitative method [3, 4]. A disadvantage is that there is a limit on the thickness of the structure being monitored [5]. A problem of signal processing in the field of active thermography methods is very important. With the help of custom developed image and signal processing algorithms, it is possible to detect small discontinuities inside structures. By reducing the amount of data, the process is accelerated, but a certain decrease in accuracy has to be kept in mind.

On the other hand, computer simulation is an economical and fast way to determine and simulate material behaviour. Finite element (FE) analysis has been applied as a verification tool in some applications involving infrared thermography $[6,7]$.

In this study, it is concluded that identical trend variations exist for both physical and analytical data with satisfactory agreement between both approaches. In this paper a method is presented to build an accurate finite element model by optimization with reduced PIRT data. The paper is further organized, starting with a motivation for the research, follewed by a description of the PIRT measurements, proceeding with the definition of the COMSOL model. Next, the optimization method based on an adaptive

*Corresponding author. Email: jeroen.peeters2@uantwerpen.be 
response surface method, described in [8] is discussed. The optimized model could be used in failure detection to assist the IR measurements and combine the benefits of surrogate modelling (fast and economical efficient) with IR NDT. It is made possible to adapt the material properties of the FE model to the realistic values of the test sample.

There are several issues that motivate the present research:

- Extensive calculation time issues for complex design models in thermography simulations. Despite the fast evolution of increasing performance of desktop PCs during the past years, complex FE models with a high number of nodes and elements still suffer from high calculation times in order to solve and combine the necessary internal equilibrium equations [8].

- The use of realistic parameter conditions improves the accuracy of FE models. With recent designs there are more and more FE models available after development. They could be reused in further maintenance and monitoring steps or for extreme condition simulations on high cost structures.

- The proposed technique will be implemented in Structural Health monitoring and NDTE (Non-destructive Testing \& Evaluation) applications. It can also be used to study environmental influences on materials.

- By updating the results with an FE model it will be possible to eliminate the time inaccuracy of microbolometer camera's.

\section{Methodology}

The proposed research is based on the Adaptive Response Surface Optimization strategy described in [8]. The algorithm is adapted for first order heat transfer problems and the combination of experimental thermographic data with FE model data. The goal is to update 7 parameters including several material specific parameters and specific experimental set-up parameters in a FE model. The resulting FE model updated with thermography simulations will produce similar results as expected from performed experimental measurements.

\subsection{Defined test case}

There is made use of a flat bottom hole plate, normally used to simulate the temperature impedance of delamination failures in composite materials. In this research the used material is a homogeneous Poly Vinyl Chloride test plate. Thereby is the chosen test case based on a bypass methodology for false positive measurements. This good thermal insulator material has isotropic distributed material properties [9] which delivers less necessary material parameters to optimize.

\subsection{Thermographic measurements}

In the following section there will be explained how the experimental measurements are performed in laboratory conditions and the used measurement technique will be described.

\subsubsection{Experimental set-up}

The PVC plate as shown in figure 1 (a) is heated by two heat sources of 1,000 W each orientated to the test plate. The sources are positioned straight to the plate as 
Table 1. Measured experimental athmospheric parameters, used tools: Testo 435.

\begin{tabular}{|l|r|l|}
\hline Condition & Value & Device \\
\hline Room Temperature $[\mathrm{deg} C]$ & 19.73 & Testo 435 \\
\hline Humidity [\%] & 37.6 & Testo 435 \\
\hline Reflected temperature [degC] & 18.1 & IR Camera and Aluminium reflection mirror \\
\hline
\end{tabular}

shown in figure 1 (b). There is made use of a Xenics Gobi 1471 microbolometric camera with a detector wavelength range of 8-14 $\mu \mathrm{m}$, a NETD (Noise Equivalent Temperature Difference) of $\geq 50 \mathrm{mK}$ and a frame size of $384 \times 288$ pixels. [10,11] The camera is placed on the centre-to-centre distance between both heat sources. The heating and cool down times are symmetric, both 75 seconds. The camera and heat sources are located on a distance of $1 \mathrm{~m}$. The atmospheric conditions are held stable and the measurements are performed in a climatic stable environment with values described in table 1.

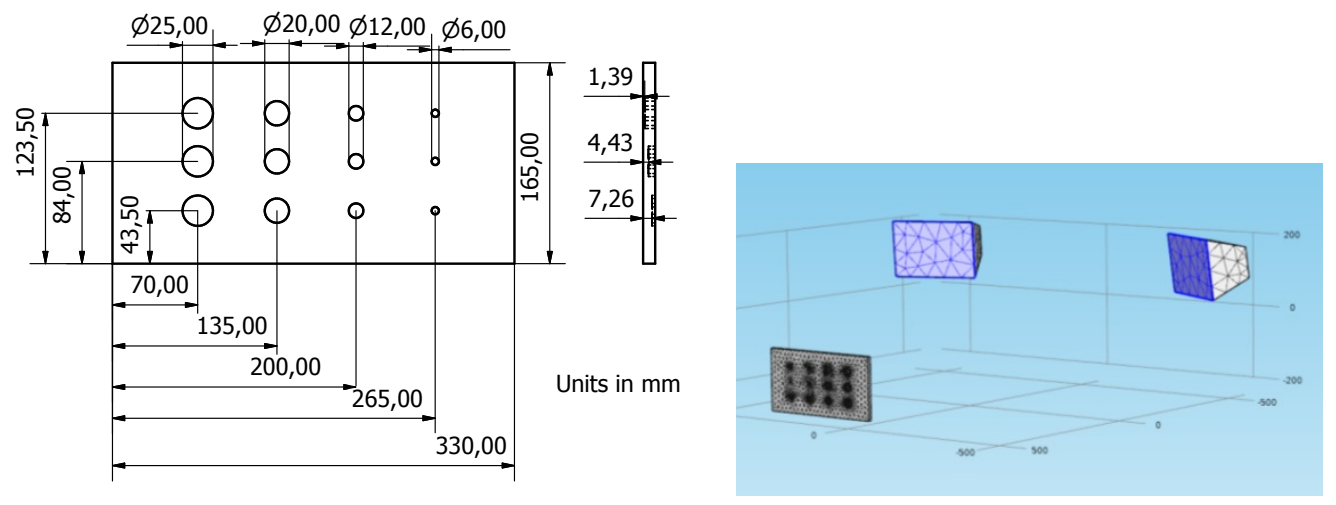

Figure 1. Left: Front and side view of the PVC plate (a) and Rigth: Experimental setup (b).

\subsubsection{Description of applied method}

The used thermographic measuring method is based on pulsed thermography (PIRT). One heat pulse with a fixed active time of 75 seconds is used to heat up the test sample resulting in a decay of approximately 10 degrees. An automatic triggering for correct timekeeping is not used in this case. There is chosen to work with uncooled cameras, based on the microbolometer principle for their good price-value ratio and to define a methodology which could be economical efficient. In general, thermographic cameras based on the principle of microbolometers don't have a fixed frame rate neither an exact trigger point. Triggering of microbolometer cameras is software based. The software decides to start measuring from the first following frame after the trigger, this is called the rolling shutter principle. Thereby there is a time offset of 1 /frame rate between the heat source and the camera. So the time inaccuracy of each frame has to be considered. Besides that, the heat sources have their own start-time, depended on the ambient temperature. This is the reason why there has been chosen to set the heat pulse trigger times as unknown, even as the effective power of the heat source.

\subsubsection{Conversion}

To decrease the amount of data and to reduce the measurement noise, the raw sensor temperature data for every frame is averaged to every second. Concerning the scope of this research, as updating the global material parameters in the FE model, this is allowed without a significant information lost. Thereby there is a decrease in frame rate fluctuation and it is made possible to compare different camera data objective in section 
3.1. For the optimization there is made use of the temperature evolution far away of the flat bottom holes as reference value and the temperature profile of the largest and deepest flat bottom hole as second target set. For the reference value there is used a dataset of nodes accordingly selected equally distributed over the test plate surface.

\subsection{Finite Element modelling}

The model is built and simulated in COMSOL Multiphysics. The governing differential equation is a pure conductive heat transfer with an external heat source, formulated in Eq. (1) where $\rho$ is the density, $C_{p}$ is the material heat capacity at constant pressure, $T$ is the absolute surface temperature, $\kappa$ is the material thermal conductivity, $t$ is the time and $Q(t)$ is the time dependent heat source [12]. The air between the lamps and the surface is neglected. The simulation set-up is shown in figure 1(b). The numerical solution can be considered sufficiently accurate for thermographic NDT applications as described in [13].

$$
\rho C_{p} \frac{\partial T}{\partial t}+\nabla \cdot(-\kappa \nabla T)=Q \text { with } T(x, y, z, 0)=T_{\text {ambient }}=292.88[K]
$$

The external heat source has a non-linear characteristic as defined in Eq. (2) where $\epsilon$ is the emissivity, $F_{\text {ambient }}$ is the field of view factor of the ambient reflections, $\sigma$ is the Boltzmann constant and $P$ is the power of the heat source [12]. There are made a few simplifications:

- The air velocity is assumed to be 0 allowed by the laboratory characteristics.

- The thermal conductivity $\kappa$ of PVC is isotropic [9].

- The test sample is opaque and behaves like an ideal grey body.

$$
Q(t)=\epsilon\left(P(t)+F_{\text {ambient }} \sigma T_{\text {ambient }}^{4}-\sigma T^{4}\right) \text { with }\left\{\begin{array}{l}
P(t)=P \text { for } t \leq 75 \\
P(t)=0 \text { for } t>75
\end{array}\right\}
$$

\subsubsection{Model characteristics}

The FE model is build of 15200 tetrahedral elements with a maximal growth rate of 1.2. The physics are designed with 2 external uniform radiating heat sources. In the simulation is assumed that the heat distribution is uniform and that the heat source housing is an ideal reflector. The power of the heat sources is multiplied with a correction coefficient for the reflection of the back of the lamp housing. The multiplication factor is in correlation with the ratio of the surface of the reflecting housing $S_{\text {reflective }}$ over the total surface of the housing $S_{\text {reflective+glass }}$ as presented in figure 2 and $\mathrm{Eq}(3)$. The temperature data points used for the optimization are placed on a grid with the same spacing as the experimental sensor grid.

$$
\frac{S_{\text {reflective }}}{S_{\text {reflective }}+S_{\text {glass }}}
$$

In reality there is some heat absorption of the heat source housing and the glass but in order of the stable atmospheric conditions, the distance between heat sources and test plate, the short pulse time and the conductive isolation of stationary air, this effect is negligible small. 

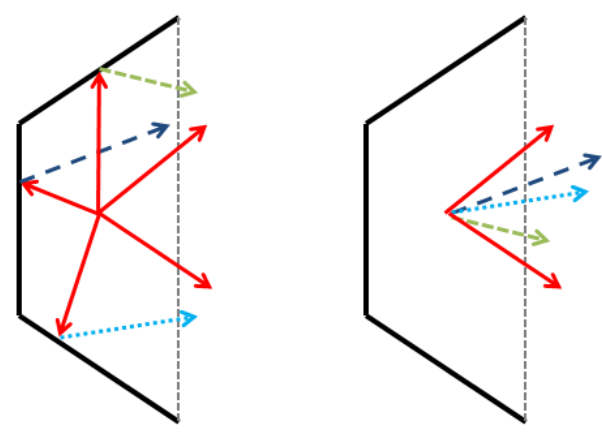

Figure 2. Schematical representation heat lamp abstraction to external heat source.

Table 2. Material Properties [9, 14-16].

\begin{tabular}{|l|r|r|}
\hline & PVC & Halogen Spots \\
\hline Thermal Conductivity $k[W /(m . K)]$ & 0.147 & 10 \\
\hline Heat Capacity $C_{p}[J /(K g . K)]$ & 1050 & 10 \\
\hline Density $\rho\left[K g / m^{3}\right]$ & 1445 & 8700 \\
\hline Surface Emissivity & 0.92 & 0.01 \\
\hline
\end{tabular}

\subsection{Optimization}

For the optimization algorithm there is made use of the adaptive response surfaces where the experimental data is used as target for the FE model. The material properties of table 2 and the experimental parameters of the pulse time, the heat power and the ambient temperature are the parameters which will be optimized as shown in table 3 . The density is excluded from the optimization parameters because it is a simple measurable, non changing parameter in industrial applications. The optimization makes use of the relative temperature differences $\theta=T-T_{0}$ to reduce the measurement uncertainties but fits it the absolute temperatures as shown in figure 6 .

The Adaptive response surface optimization algorithm is used to optimize numerical models with a large amount of data points and the time reducing by the algorithm increases as the amount of parameters rises [17]. The optimization procedure could be divided in a certain steps:

(1) Run starting reference simulation points and building a correct object function of the difference between the FE model and the target value (experiment or validation model).

(2) Replace the FE model by a meta model of response surfaces to decrease the optimization time.

(3) Run the optimization routine on a well-defined object function. In this paper, the object function is weighted to increase the differences between the different measurement points (pixels) that are taken into account.

(4) Use the estimated parameter values as input parameters for a new FE model that corrects the response model.

(5) Only the points the closest to the minimum will be used to form the response surface.

The response model to be optimized is not built from a pre-defined number of design experiments but is adapted and refined during the optimization routine by a pan and zoom command [8]. 


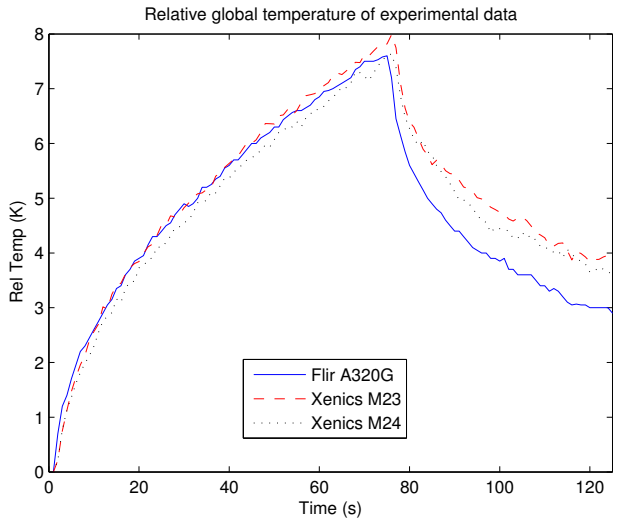

Figure 3. Relative temperature for different camera's in function of the corrected time.

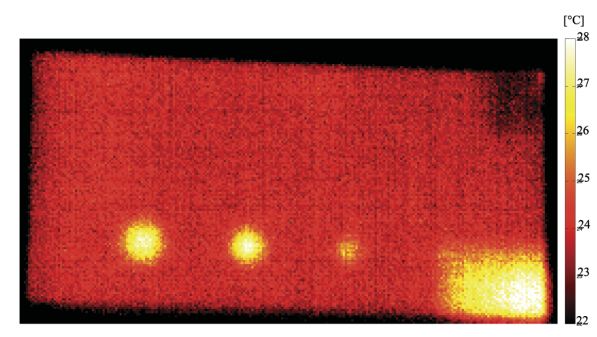

Figure 4. Thermal result of Xenics camera after $75 \mathrm{sec}$ of heating.

\section{Results}

In a first stage the experimental values are compared between 2 independent measurements with the Xenics camera and one measurement of a Flir A320G device. Besides, the optimization algorithm is validated between $2 \mathrm{FE}$ models to check the convergence to a global minimum with different optimization strategies. And in a last part there is made a comparison between different optimization strategies with the experimental target input.

\subsection{Experimental results}

The experimental measurements from the Xenics camera are verified with a Flir A320G device with comparable specifications and autofocus, specified in [18]. The absolute temperature uncertainty is $\pm 2 K$. The target value of the optimization is an averaging of 2 independent measurements of the Xenics camera. To compare the data and to increase the accuracy, the measure points are interpolated between the different frames and averaged to $1 \mathrm{~Hz}$ as explained in section 2.2.3 and there is worked with the relative temperature between the time step and $T_{\text {ambient }}$. The results are shown in figure 3 . And as could be seen, the increasing flank of the heat pulse is sufficiently coherent between the different measurements. Unfortunately there could be seen that the error between the Flir camera and the Xenics camera increases on the negative flank. The reason for this could be found in the fluctuating frame rate of both cameras but this should be verified. In figure 4 is the thermal result shown after $75 \mathrm{sec}$. The rectangular heat spot on the bottom right is the reflection of one of the heat sources and is excluded from the further analysis. The more interesting heat spots are the deepest flat bottom holes in the plate.

\subsection{Optimization results}

In a first stage the optimization algorithms are validated between $2 \mathrm{FE}$ models to check for convergence and accuracy. In the following subsections the different recursive models will be discussed.

There is made use of a multidimensional piecewise polynomial fitting, based on [8]. For all time steps, there is made a regressive polynomial model of the relative temperature of the plate surface versus the different optimization parameters. The parameters to optimize for are defined in table 3 and chosen accordantly with the measurement boundaries in literature. The real values are described in table 2 . The mass density is excluded from 
Table 3. Optimization parameters with their boundary conditions [14].

\begin{tabular}{|l|r|r|}
\hline Parameter & Lower boundary & upper boundary \\
\hline Heat source power $[W]$ & 800 & 1000 \\
\hline Time until start of the heat pulse $[s]$ & 0 & 10 \\
\hline Time of the heat pulse $[s]$ & 70 & 80 \\
\hline Ambient temperature $[K]$ & 280 & 300 \\
\hline Thermal conductivity $k[W /(m . K)]$ & 0.12 & 0.209 \\
\hline Specific heat capacity $C_{p}[J /(K g . K)]$ & 1000 & 1800 \\
\hline Emissivity & 0.90 & 0.98 \\
\hline
\end{tabular}

the optimization parameters to avoid interference with the specific heat capacity and for reasons explained in 2.4 .

There is made use of 2 different optimization solvers, a non-linear least squares solver (lsqnonlin) and a least-squares curve fitting solver for data fitting purposes (lsqcurvefit). Within both solvers, both algorithms, the Levenberg-Marquardt (LM) and the trustregion-regression (TRR) are compared [19]. The object function of both solvers is formed by the comparison between the FE model and the target values from the experiments for at least five points and can be extrapolated for all points. In the objective function of the lsqnonlin solve there is made use of some extra conditions to cause convergence. This extra conditions are based on post processing data extracted of the experimental data to retrieve the correct pulse times and ambient temperature. There is made use of weighting factors in the lsqnonlin solver to improve the converged result between the several objective functions.

\subsubsection{Model validation}

To verify the proposed methodology on a heat transfer model and the choice of estimated parameters, the first step was to switch the unknown experimental target value with a well-known FE validation model. This simulation has a fixed value for all the estimated parameters and could be compared with a perfectly controlled, noiseless experimental measurement. The methodology could be verified by running the defined optimization algorithm with this fixed simulation as target value. The results of the validation routine are mixed and strongly dependent on the option values of the optimization algorithms and the choice of parameters. In this paper there is studied after the effects on dependent parameters as the heat source power and pulse times or $C_{p}$ and $k$. To retrieve a sufficiently accurate value of all the 7 parameters, there should be looked after the most ideal option values to calibrate the methodology. The results are defined in table 4 . Further on there is made a comparison with an optimization without the ARSM method which solves a changed FE-model each time the optimization solver makes an iteration. As could be seen in table 4, reasonable approximation results are acquired for 6 of the 7 parameters. The reason that the specific heat does not converge to the correct value is unknown. There could be suggested that it strongly depends on the influence of that specific parameter on the response of the system. For example, an increase of $50 \%$ of the thermal conductivity results in an increase of $50 \%$ of the thermal diffusivity. But a same increase of the specific heat capacity or the density would result in a decrease of $33 \%$. Further on both parameters are dependent on each other which results in a conflict of importance. This combined with the influence of all the other parameters may result in a neglectable importance of the specific heat capacity parameter in the simulation model by polynomial approximation. Remarkable is that especially the LM algorithm does not have the same problem with the dependent parameters of the heat source. These approximations should be investigated more in detail in the future.

Globally there can be concluded that the Levenberg-Marquardt algorithm delivers better results than the trust-region-reflective but needs more optimization runs in order 
Table 4. Optimization results model validation.

\begin{tabular}{|c|c|c|c|c|c|c|}
\hline Description & Target & $\begin{array}{r}\text { FE } \\
\text { optimization }\end{array}$ & $\begin{array}{c}\text { Polynom } \\
\text { lsqnonlin } \\
\text { LM }\end{array}$ & $\begin{array}{r}\text { Polynom } \\
\text { lsqnonlin } \\
\text { TRR }\end{array}$ & $\begin{array}{r}\text { Polynom } \\
\text { lsqcurvefit } \\
\mathrm{LM}\end{array}$ & $\begin{array}{r}\text { Polynom } \\
\text { lsqcurvefit } \\
\text { TRR }\end{array}$ \\
\hline Heat source power [W] & 920.00 & 919.94 & 922.22 & 958.76 & 916.22 & 946.87 \\
\hline Time left $[\mathrm{s}]$ & 0.5000 & 0.5000 & 0.19165 & 0.50163 & 0.87050 & 1.2514 \\
\hline Time right $[\mathrm{s}]$ & 75.500 & 75.504 & 75.528 & 75.453 & 75.505 & 75.641 \\
\hline$T_{\text {ambient }}[\mathrm{K}]$ & 293.15 & 293.15 & 293.16 & 293.09 & 293.15 & 293.21 \\
\hline $\mathrm{k}[\mathrm{W} /(\mathrm{m} \cdot \mathrm{K})]$ & 0.14700 & 0.14671 & 0.14703 & 0.15230 & 0.14738 & 0.14190 \\
\hline$C_{p}[\mathrm{~J} /(\mathrm{Kg} . \mathrm{K})]$ & 1050.0 & 1598.6 & 1602.3 & 1727.5 & 1604.1 & 1542.4 \\
\hline$\epsilon$ & 0.92000 & 0.92000 & 0.91445 & 0.94800 & 0.92622 & 0.91169 \\
\hline Optimization runs & 1 & 5 & 21 & 13 & 18 & 10 \\
\hline Optimization time [sec] & / & 74886 & $\begin{array}{l}5304.4 \\
(3204.4)\end{array}$ & $\begin{array}{r}5062.6 \\
(2962.6)\end{array}$ & $\begin{array}{r}5259.3 \\
(3159.3)\end{array}$ & $\begin{array}{r}5092.2 \\
(2992.2)\end{array}$ \\
\hline rms residual & l & 0.24693 & 3.3549 & 14.296 & 0.62542 & 7.0650 \\
\hline
\end{tabular}

to converge as can be seen in figure 5. A second result can be that the lsqcurvefit delivers better results than the lsqnonlin solver. Side mark to this results is that the lsqnonlin solver needs accurate weighting factors which results in an unstable result. The lsqcurvefit solver delivers more stable results without weighting factors in less optimization runs. The most accurate results are found with the FE optimization without the ARSM method and this in the fewest optimization runs. By comparison of the optimization times should be mentioned that the times of the polynomial optimization implements the calculation time of the 12 starting points as described in point one of section 2.4. The used time for the starting points is the same for all polynomial optimizations and is around $2100.0 \mathrm{sec}$. Thereby the actual optimization time is mentioned in brackets in table 4. Out of this data we could conclude that the ARSM method results in a 25 times faster optimization than the FE optimization. And by using the lsqcurvefit solver with the Levenberg-Marquardt (LM) algorithm the residual value is just slidely more than the FE optimization routine.

There could be concluded that the adaptive response optimization delivers a faster result but that the convergence and accuracy strongly depends on the option settings.

\subsubsection{Regresive optimization with polynomials of experimental target values}

The experimental target values are compared with FE model data placed on a grid, accordantly with the pixel grid of the camera. In a first stage there is made use of an objective function based on 7 points, the three centres of the largest flat bottom holes and 4 reference points distributed following the first diagonal from the left bottom to the right upper corner, between the flat bottom holes. The objective functions are build on the same way as mentioned in section 3.2.1. The results and accuracy of the different optimization methods are shown in table 5 . As could be seen in table 5 the lsqnonlin optimization delivers a result in less than a half of the optimization steps from the lsqcurvefit. But is the total optimization time of the lsqcurvefit functions hardly more to retrieve a smaller root-mean-squared residual value. In figure 6 is compared the time plot of the temperature profile for the largest defect of figure 4 for the measurements and the approximations. There could be seen that the lsqcurvefit approximation delivers the best results for the decay and the time positioning of the maximum temperature point. Only the height of the maximum temperature has some overshoot which is less for the LM algorithm than for the TRR. 


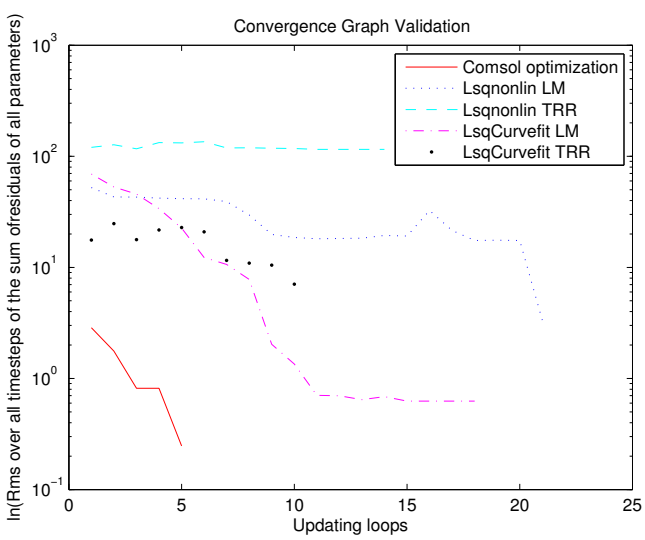

Figure 5. Convergence of the object functions of the validation case.

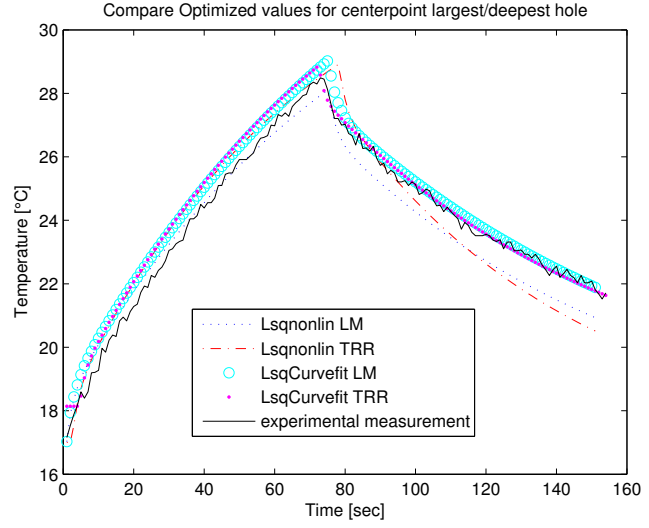

Figure 6. Evaluation of the approximation of the FE model to the experimental model for specific points.

Table 5. Optimization results experimental set-up by polynomial regression model.

\begin{tabular}{|c|c|c|c|c|c|}
\hline Description & Target & $\begin{array}{c}\text { Polynom } \\
\text { lsqnonlin } \\
\text { LM }\end{array}$ & $\begin{array}{r}\text { Polynom } \\
\text { lsqnonlin } \\
\text { TRR }\end{array}$ & $\begin{array}{r}\text { Polynom } \\
\text { lsqcurvefit } \\
\mathrm{LM}\end{array}$ & $\begin{array}{r}\text { Polynom } \\
\text { lsqcurvefit } \\
T R R\end{array}$ \\
\hline Heat source power [W] & [850-1000] & 920.47 & 923.84 & 901.40 & 951.05 \\
\hline Time left $[\mathrm{s}]$ & {$[2.0-3.0]$} & 0.48272 & 2.5816 & 6.1040 & 4.7460 \\
\hline Time right $[\mathrm{s}]$ & {$[72.0-73.0]$} & 73.328 & 78.459 & 71.436 & 72.666 \\
\hline$T_{\text {ambient }}[\mathrm{K}]$ & [291.55-291.15] & 291.97 & 291.55 & 291.39 & 291.29 \\
\hline $\mathrm{k}[\mathrm{W} /(\mathrm{m} \cdot \mathrm{K})]$ & {$[0.12-0.209]$} & 0.084509 & 0.082792 & 0.13550 & 0.13583 \\
\hline$C_{p}[\mathrm{~J} /(\mathrm{Kg} . \mathrm{K})]$ & [1000-1800] & 825.77 & 1000.0 & 833.11 & 1000.0 \\
\hline$\epsilon$ & {$[0.94-0.96]$} & 0.91798 & 0.92377 & 1.0125 & 0.98871 \\
\hline Optimization runs & / & 10 & 7 & 23 & 25 \\
\hline Optimization time & / & $\begin{array}{r}5649.5 \\
(3549.5)\end{array}$ & $\begin{array}{r}3291.0 \\
(1191.0)\end{array}$ & $\begin{array}{r}5729.0 \\
(3629.0)\end{array}$ & $\begin{array}{r}5964.3 \\
(3864.3)\end{array}$ \\
\hline rms residual & 1 & 434.00 & 519.62 & 422.67 & 421.44 \\
\hline
\end{tabular}

\section{Conclusions}

In this paper, a new adaptive method is described to update the material parameters of an FE model by pulsed thermography measurements. There is a large optimization time decrease by using an adaptive regressive model of the FE model. The importance of the time decrease rises with the amount of parameters used in the optimization routine. With this method it is possible to validate an FE model with experimental results and adapt it properly. This delivers a large amount of possible applications where the benefits of thermography and FE modelling could be combined. The best and fastest results are achieved by using a lsqcurvefit solver and the Levenberg-Marquardt algorithm. This delivers results which are independent of weighting factors and the strategy is robust for some dependency between parameters. Unfortunately not all parameters are approximated perfectly and the residual value is dependent on the noise in the signal but the global result for each pixel retrieves promising accordance.

\section{Acknowledgements}

This research has been funded by the University of Antwerp and the Institute for the Promotion of Innovation by Science and Technology in Flanders (IWT). Furthermore, the research leading to these results has received funding from Industrial Research Fund FWO Krediet aan navorsers 1.5.240.13N. The authors also acknowledge the Fund for 
Scientific Research Flanders (FWO), the Flemish government (GOA-Optimech) and the research councils of the Vrije Universiteit Brussel (OZR) and University of Antwerp (fti-OZC) for their funding. I would like to thank Prof.Dr.Ir. Luc Mertens for software support and equipment.

\section{References}

[1] Vollmer M, Möllmann KP. Infrared Thermal Imaging. Wiley-VCH, Berlin; 2011.

[2] Susa M, Ibarra-Castanedo C. Pulse thermography applied on a complex structure sample: comparison and analysis of numerical and experimental results. IV Pan Am .... 2007; Available from: http://212.8.206.21/article/panndt2007/papers/54.pdf.

[3] Ibarra-Castanedo C, Genest M, Piau JM, Guibert S, Bendada A, Maldague XPV. Active Infrared Thermography Techniques for the Non-destructive Testing of Materials. In: Ultrason. adv. methods nondestruct. test. mater. charact. Chen, CH; 2007. p. 325-348; Available from: http://medcontent.metapress.com/index/A65RM03P4874243N.pdf.

[4] Giorleo G, Meola C. Comparison between pulsed and modulated thermography in glassepoxy laminates. NDT E Int. 2002;35:287-292; Available from: http://www.sciencedirect.com/science/article/pii/S0963869501000627.

[5] Busse G. Techniques of Infrared Thermography: Part 4. Lock-in thermography. In: Nondestruct. handb. 3rd ed. Columbus, Ohio: ASNT Press; 2001. p. 718.

[6] Louaayou M, Naït-Saïd N, Louai FZ. 2D finite element method study of the stimulation induction heating in synchronic thermography NDT. NDT E Int. 2008 Dec;41(8):577-581; Available from: http://linkinghub.elsevier.com/retrieve/pii/S096386950800073X.

[7] Chaudhuri P, Santra P, Yoele S, Prakash A, Reddy D, Lachhvani L, Govindarajan J, Saxena Y. Non-destructive evaluation of brazed joints between cooling tube and heat sink by IR thermography and its verification using FE analysis. NDT E Int. 2006 Mar;39(2):88-95; Available from: http://linkinghub.elsevier.com/retrieve/pii/S0963869505001167.

[8] Steenackers G, Presezniak F, Guillaume P. Development of an adaptive response surface method for optimization of computation-intensive models. Comput Ind Eng. 2009 Oct;57(3):847-855; Available from: http://linkinghub.elsevier.com/retrieve/pii/S0360835209000825.

[9] Dashora P, Gupta G, Dashora J. Thermal conductivity, diffusivity and heat capacity of plasticized polyvinyl chloride. Indian J Pure .... 2005;43(February):132-136; Available from: http://nopr.niscair.res.in/handle/123456789/8720.

[10] (Xenics Vision). Sensor-Gobi384-1471. 2010.

[11] (Xenics Vision), Uwaerts D. Gobi Interface Control Document XP Gobi 384-1471. 2008.

[12] Chapman AJ. Heat transfer. 4th ed. New York: Macmillan; 1984.

[13] Darabi A, Maldague X. Neural network based defect detection and depth estimation in TNDE. NDT E Int. 2002;Available from: http://ac.elscdn.com/S096386950100041X/1-s2.0-S096386950100041X-main.pdf?_tid=48a5f6229d30-11e3-b900-00000aacb360\&acdnat=1393231825_c8c438208474ca17a066479cb5d67f35 http://www.sciencedirect.com/science/article/pii/S096386950100041X.

[14] Granta. PVC semi-rigid, molding and extrusion. 2013.

[15] (professional thermographers association). Emissivity Values for Common Materials. 2014; Available from: http://www.infrared-thermography.com/material.htm.

[16] Carcangiu S, Cannas B, Concu G, Trulli N. Modeling of Active Infrared Thermography for Defect Detection in Concrete Structures. In: comsol Conf. Milan. Milan: Comsol; 2012. p. 7; Available from: http://www.comsol.pt/conference2012/europe/abstract/id/13627/carcangiu_abstract.pdf.

[17] Steenackers G, Guillaume P. Finite element model updating taking into account the uncertainty on the modal parameters estimates. J Sound Vib. 2006 Oct;296(4-5):919-934; Available from: http://linkinghub.elsevier.com/retrieve/pii/S0022460X06002719.

[18] FLIR. The Ultimate Infrared Handbook for R \& D Professionals. 2013.

[19] The MathWorks. The Matlab user manual. 2013. 